\title{
AZD8055 induces cell death associated with autophagy and activation of AMPK in hepatocellular carcinoma
}

\author{
MIN HU $^{1 *}$, HAILI HUANG ${ }^{1 *}$, RUI ZHAO ${ }^{3}$, PENG LI $^{2}$, MINGYI LI $^{1}$, \\ HUILAI MIAO ${ }^{1}$, NIANPING CHEN ${ }^{1}$ and MING CHEN ${ }^{1}$ \\ ${ }^{1}$ Laboratory of Hepatobiliary Surgery, ${ }^{2}$ Clinical Research Center, Affiliated Hospital of Guangdong \\ Medical College, Zhanjiang, Guangdong 524001; ${ }^{3}$ Institute of Genetic Engineering, \\ Southern Medical University, Guangzhou, Guangdong 510515, P.R. China
}

Received July 8, 2013; Accepted September 7, 2013

DOI: $10.3892 / o r .2013 .2890$

\begin{abstract}
AZD8055 is a potent inhibitor of mTORC1 and mTOR2 and shows inhibitory effects in several types of cancer cells in vitro and in vivo. However, the effect of AZD8055 on hepatocellular carcinoma (HCC) cells has not been studied. We report that AZD8055 inhibits cell proliferation and colony formation of Hep3B and Huh7 cells but does not cause PARP cleavage, or caspase activation, suggesting that classical apoptosis is not its main mechanism of cell death. By contrast, AZD8055-induced cell death was associated with several characteristics of autophagy, including an increase in acidic vesicular organelle content, conversion of cytosolic LC3-I to membrane-bound LC3-II and elevation of the levels of Atg-5/12, BECN1 and LC3-II. Inhibition of autophagy by 3-methyladenine (3-MA) partially inhibited AZD8055-induced cell death. Furthermore, AZD8055 caused the activation of AMPK and co-treatment with the AMPK inhibitor dorsomorphin also caused a partial but significant reduction of AZD8055-induced cell death. In conclusion, AZD8055-induced HCC cell death is associated with induction of autophagy and activation of AMPK.
\end{abstract}

\section{Introduction}

Hepatocellular carcinoma (HCC) is one of the most common malignant tumors and ranks fifth in frequency worldwide among all malignancies; it causes 1 million deaths annually and its incidence is increasing steadily in various countries (1). Epidemiological studies showed that primary liver cancer is the second cause of cancer-related mortality in China and it accounts for $53 \%$ of all liver cancer deaths worldwide (2).

Correspondence to: Professor Mingyi Li, Laboratory of Hepatobiliary Surgery, Affiliated Hospital of Guangdong Medical College, Zhanjiang, Guangdong 524001, P.R. China

E-mail: limingyi63@163.com

*Contributed equally

Key words: AZD8055, autophagy, hepatocellular carcinoma, AMPK
Despite considerable developments in diagnosis and therapy, the cure rate for patients who undergo resection is relatively low, and among patients who are ineligible for surgical or percutaneous procedures, only chemoembolization improves survival. Moreover, HCC is widely regarded as a chemotherapy-resistant disease. These drawbacks necessitate the continued search for novel HCC therapies.

AZD8055 is an orally available, potent inhibitor of mTORC1 and mTOR2 with superior pharmacokinetic and activity profiles and shows inhibitory effects in a wide variety of tumor cells by inhibiting the phosphorylation of mTORC1 substrates p70S6K and 4E-BP1 as well as phosphorylation of the mTORC2 substrate AKT and downstream proteins $(3,4)$. Combination of AZD8055 and MEK (Selumetinib) inhibitor enhanced apoptosis and tumor growth suppression in non-small cell lung cancer (5). The promising results of AZD8055 demonstrate its potential as a therapeutic agent for solid tumors individually or in combination, and warrant our investigation into the therapeutic potential and molecular mechanism of AZD8055 in liver cancer cells.

Autophagy, a process of 'self-eating', is a process of selfdegradation that maintains cellular viability during periods of metabolic stress (6). Although autophagy is considered a survival mechanism when faced with cellular stress, extensive autophagy can also lead to cell death. Accumulating evidence indicates the crucial role of autophagy as a key molecular mechanism to eliminate cancer cells in chemotherapy. AZD8055 has been shown to induce autophagy in several cancer cell lines, although there are controversies if autophagy induced by AZD8055 serves a pro-death or a pro-survival role (3-5,7-9).

In the present study, we focused on the therapeutic value and molecular mechanism of AZD8055 in HCC cells. We showed that AZD8055 induces cell death associated with autophagic features including an increase in the number of acidic vacuole organelles and conversion of cytosolic LC3-I to membrane-bound LC3-II. Furthermore, AZD8055 induced the activation of AMPK and co-treament with AMPK inhibitor dorsomorphin attenuated AZD8055-induced cell death. These findings provide a better understanding of AZD8055-induced cell death and demonstrate the potential of AZD8055 as a therapeutic agent for HCC; they also provide insight into the development of novel therapies for HCC. 


\section{Materials and methods}

Chemicals and antibodies. AZD8055 was purchased from Selleck Chemicals, dissolved in dimethyl sulfoxide (DMSO) as a stock concentration of $1 \mathrm{mmol} / \mathrm{l}$ and diluted to the indicated concentrations with culture medium. All antibodies were obtained from Cell Signaling Technology. Cell culture media and supplements were purchased from Gibco/Invitrogen Corp. Other chemicals and reagents were obtained from Sigma-Aldrich.

Cell lines and cell culture. Human HCC cell lines Huh7 and Hep3B were obtained from the American Type Culture Collection. Cell lines were maintained in RPMI-1640 medium with $10 \%$ FBS supplemented with $100 \mathrm{U} / \mathrm{ml}$ penicillin $\mathrm{G}$ and $100 \mu \mathrm{g} / \mathrm{ml}$ streptomycin (Sigma-Aldrich). Cells were maintained at $37^{\circ} \mathrm{C}$ in a humidified $5 \% \mathrm{CO}_{2}$ incubator.

Cell viability and cell death assay. Huh7 and Hep3B cells $\left(1 \times 10^{5} / \mathrm{ml}\right)$ were plated in 96-well plates and allowed to attach overnight. Cells were exposed to various concentrations of AZD8055 for the indicated times. Cell viability was assessed by the reduction of tetrazolium bromide (MTT) assay as previously described (10). For assay of cell death, cells were treated with AZD8055 at different concentrations for the indicated times. After treatment, cell death was determined by trypan blue exclusion as previously described (11). Briefly, cells were stained with $0.4 \%$ trypan blue and stained cells were counted. Data represent the mean \pm SD derived from at least 3 separate experiments.

Clonogenicity assay. Clonogenicity assay was performed as previously described (12). Briefly, Huh7 and Hep3B cells were plated in 6-well plates in RPMI-1640 media containing $100 \mathrm{nM}$ AZD8055 and colonies were stained with crystal violet and counted in triplicate wells after growth for a further 2 to 3 weeks. DMSO was used as a negative control. Data represent the mean \pm SD derived from at least 3 separate experiments.

Immunofluorescent staining. Cells were fixed in $4 \%$ paraformaldehyde and blocked with normal goat serum. The mouse anti-human LC3 (Cell Signaling Technology) primary antibody was added and incubated overnight at $4^{\circ} \mathrm{C}$. After washing 3 times with PBS, the goat anti-mouse IgG secondary antibodies conjugated with Cy3 (Jackson ImmunoResearch Laboratories Inc., West Grove, PA, USA) were added and incubated at room temperature for $1 \mathrm{~h}$. Cells were then counterstained with DAPI (Sigma-Aldrich) and the images were captured using a Leica fluorescent microscope.

Immunoblotting. Immunoblotting was performed as previously described (10). At the end of the treatments, Huh7 cells were harvested and lysed with ice-cold cell lysis solution and the homogenate was centrifuged at $10,000 \mathrm{x}$ g for $15 \mathrm{~min}$ at $4^{\circ} \mathrm{C}$. Total protein in the supernatant was quantified using a BCA protein assay kit. Total protein $(30 \mu \mathrm{g})$ from each sample was separated by $12 \%$ SDS-PAGE and transferred to a PVDF membrane; the PVDF membrane was placed in washing buffer containing skimmed milk powder at room temperature and blocked for $2 \mathrm{~h}$. After washing 3 times, indicated primary antibodies were added respectively and incubated at $4^{\circ} \mathrm{C}$ over- night. Then, horseradish peroxidase-conjugated secondary antibody was added to incubate for $1 \mathrm{~h}$. X-ray film exposure was performed and AlphaImager HP fluorescence/visible light gel imaging analyzer processing and image analysis software were used to analyze gray value.

Statistical analysis. All data are presented as the mean \pm SD. Differences between groups were analyzed for statistical significance using a two-tailed Student's t-test. $\mathrm{P}<0.05$ was considered to indicate statistically significant differences.

\section{Results}

AZD8055-induced cell death is associated with caspasedependent apoptotic signaling cascade. To study the cytotoxic effect of AZD8055 on HCC cells, Huh7 and Hep3B cells were used in the present study. Initially, we determined the effects of AZD8055 on cell death in both cell lines which were measured by trypan blue exclusion. DMSO was used as control. Huh7 and Hep3B cells were treated with different concentrations $(5,10$, 20,50 and $100 \mathrm{nM}$ ) of AZD8055 $24 \mathrm{~h}$ or with $100 \mathrm{nM}$ AZD8055 for various periods and trypan blue staining was performed. As shown in Fig. 1A, AZD8055 increased cell death in both cell lines in a concentration- and time-dependent manner. AZD8055 $(100 \mathrm{nM})$ was used in the following study, which was shown to significantly induce cell death in both cell lines.

To determine the long-term cytotoxicity of AZD8055, colony formation assay was performed in Huh7 and Hep3B cells treated with $100 \mathrm{nM}$ AZD8055 or DMSO. As shown in Fig. 1B, AZD8055 decreased colony numbers in both cell lines, as compared to control cells. These data indicate that AZD8055 is cytotoxic to HCC cells.

To investigate the nature of AZD8055-induced cytotoxicity, we first determined whether AZD8055 inhibits HCC cell proliferation through classical apoptotic type I cell death. Huh7 and Hep3B cells were treated with DMSO or $100 \mathrm{nM}$ AZD8055 for $24 \mathrm{~h}$. We explored whether AZD8055induced cell death was associated with caspase activation. Immunoblot analysis revealed that AZD8055 treatment did not markedly induce the formation of the active forms of cleaved caspase-3 and caspase-9. Consistently, the cleavage of PARP, which is a substrate of caspase-3 and a well-known apoptotic hallmark, was not upregulated by AZD8055 in tested cell lines. Quantitative analysis of caspase-3, caspase-8 and caspase- 9 activity by colorimetric assay also showed that AZD8055 treatment did not alter caspase-3, caspase-8 and caspase-9 activity in the tested cell lines. These data suggest that AZD8055-induced cell death is not associated with the caspase-dependent apoptotic signaling cascade.

AZD8055 induces autophagy in HCC cells. Since AZD8055induced cell death appeared to lack the characteristics of classical apoptotic type I cell death, we sought to determine whether it was associated with changes in the autophagic pathway previously described as characteristic of type II cell death. To determine whether AZD8055 induces autophagy in HCC cells, we investigated the effect of the drug on the intracellular localization of microtubule-associated protein 1 light chain 3 (LC3), a specific marker of autophagosomes $(13,14)$, in the tested cell lines. As shown in Fig. 2A, left, representa- 

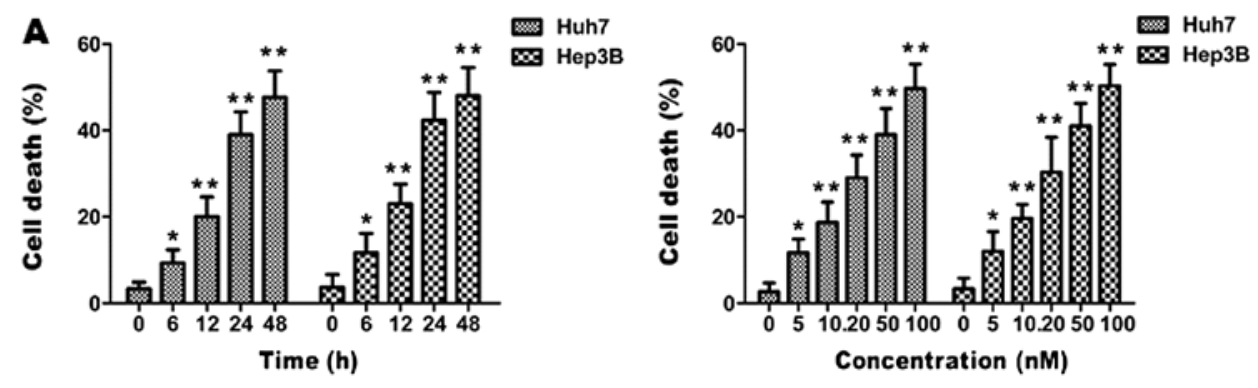

B

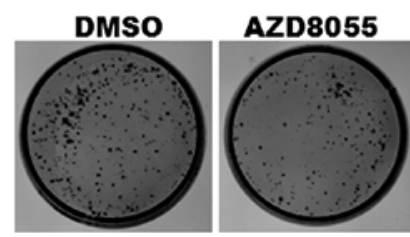

Huh7

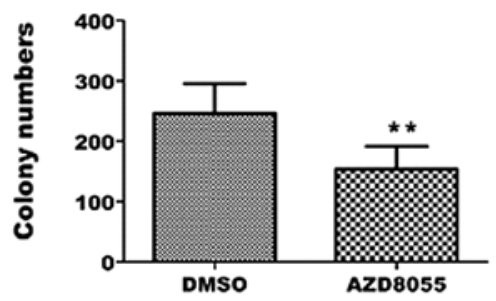

C
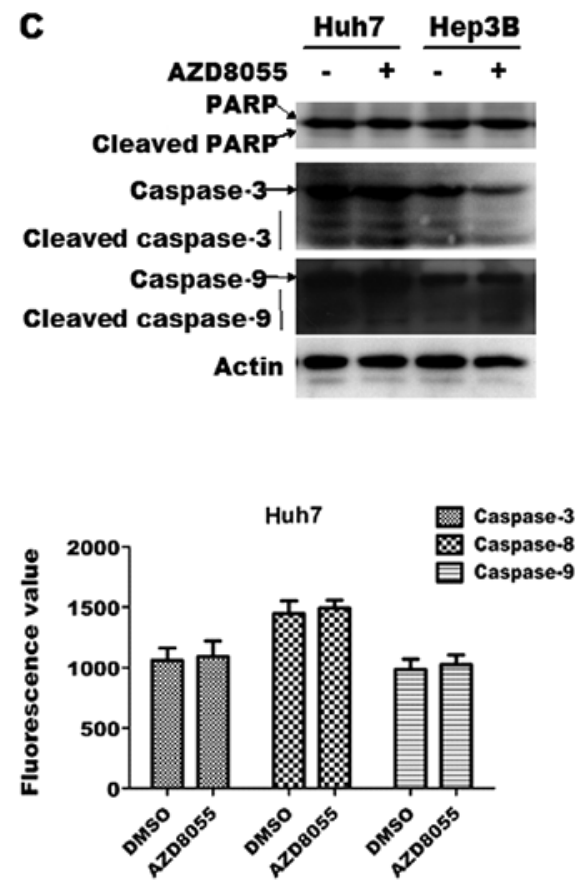

DMSO AZD8055

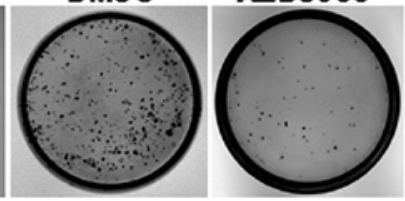

Hep3B
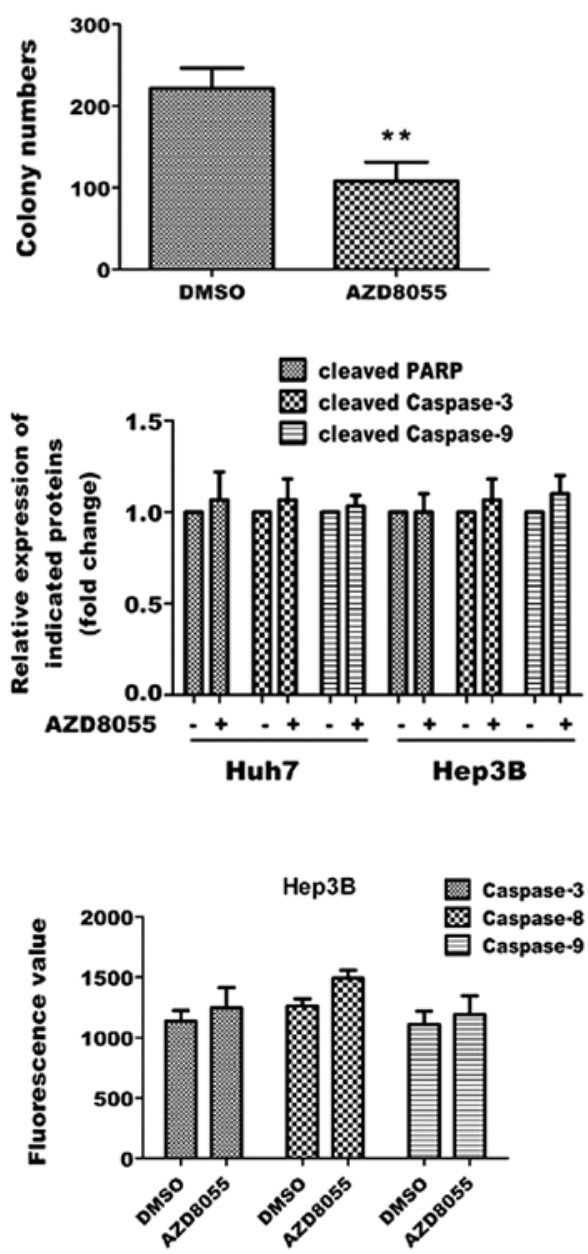

Figure 1. AZD8055-induced cell death is associated with caspase-dependent apoptotic signaling cascade. (A) AZD8055 induces cell death in a time- and concentration-dependent manner. Huh7 and Hep3B cells were treated with different concentrations (5, 10, 20, 50 and 100 nM) of AZD8055 for 24 h or were treated with $100 \mathrm{nM}$ AZD8055 for variable periods (6, 12, 24 and $48 \mathrm{~h})$. Cell death was assessed by performing MTT assay and trypan blue staining, respectively. Data represent the mean \pm SD derived from at least 3 separate experiments. ${ }^{*} \mathrm{P}<0.05,{ }^{* *} \mathrm{P}<0.01$, compared to control. (B) AZD8055 suppresses colony formation of HCC cells. Huh7 and Hep3B cells were plated in 6-well plates in RPMI-1640 media containing 100 nM AZD8055 and colonies were stained with crystal violet and counted in triplicate wells after growth for a further 2 to 3 weeks. DMSO was used as control. Data represent the mean \pm SD derived from at least 3 separate experiments. ${ }^{* *} \mathrm{P}<0.01$. (C) AZD8055 does not cause caspase activation. Huh7 and Hep3B cells were treated with $100 \mathrm{nM}$ AZD8055 for $24 \mathrm{~h}$ and immunoblot analysis was performed to detect PARP cleavage and caspase-3, caspase-9 activation. $\beta$-actin was used as a loading control (up). Quantitative analysis of caspase-3, caspase- 8 and caspase- 9 activity was assessed by colorimetric assay (down). Data represent the mean \pm SD derived from at least 3 separate experiments.

tive fluorescence micrographs show a punctate pattern of LC3 in AZD8055-treated cells in contrast to the diffuse pattern in control cells. Quantitative analysis revealed a significant increase in cells with $\mathrm{LC} 3$ punctate pattern in AZD8055-treated cells as compared with control cells $(\mathrm{P}<0.01)$, i.e., 43 vs. $17 \%$ in Huh7 cells and 41 vs. 18\% in Hep3B cells, respectively (right). 

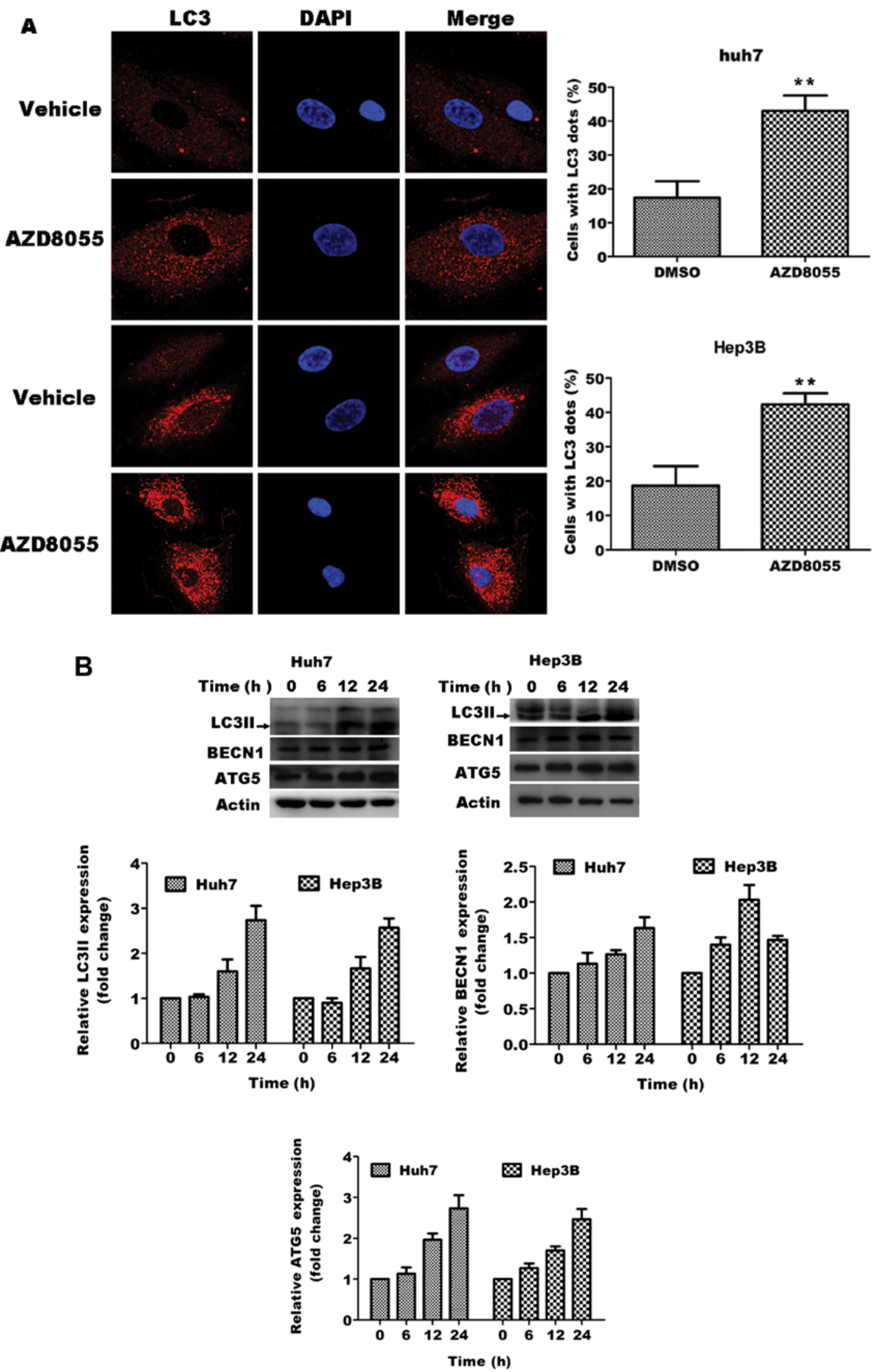

Figure 2. AZD8055 induces autophagy in HCC cells. (A) Effect of AZD8055 on intracellular localization of LC3 protein. Huh7 cells were treated with $100 \mathrm{nM}$ AZD8055 or with the same volume of DMSO as control for $12 \mathrm{~h}$. Immunofluorescence staining was performed to examine the transformation of cytosolic LC3-I to membrane-bound LC3-II. Quantitatively, percentage of cells with dots of LC3 in control or AZD8055-treated cells were assessed by counting at least 100 cells under fluorescence microscopy (right). Each column represents mean \pm SD of 3 independent experiments. ${ }^{* *} \mathrm{P}<0.01$. (B) Effect of AZD8055 on the expression of Atg-5/12, BECN1 and LC3-II in human HCC cell lines. Huh7 cells were treated with $100 \mathrm{nM}$ AZD8055 for the indicated times. After treatment, cell extracts were prepared for immunoblot analysis. The levels of Atg-5/12, BECN1 and LC3-II were detected using the corresponding antibodies. $\beta$-actin was used as a loading control (left). The amount of Atg-5/12, BECN1 and LC3-II was quantified by a laser densitometer. After normalization to $\beta$-actin, the relative levels of Atg-5, BECN1 and LC3-II were compared with those at time 0 as a value of one. Data represent mean \pm SD of 3 independent experiments (right). 
A
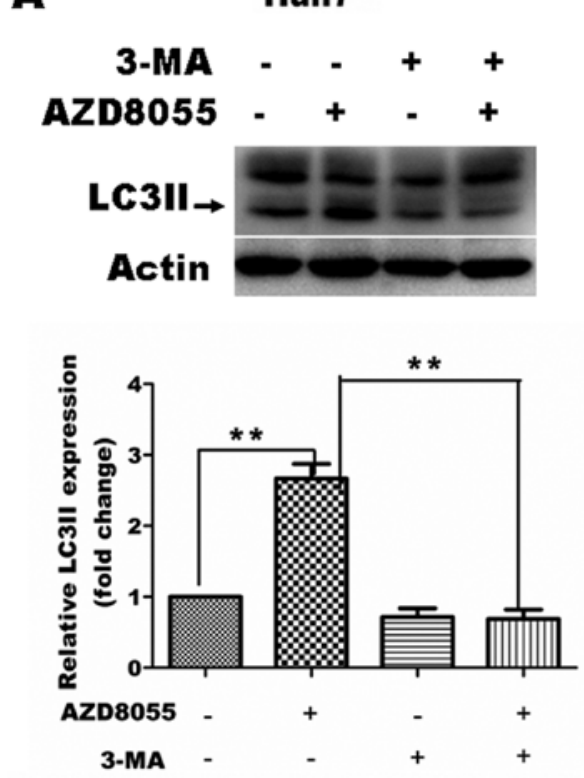

B

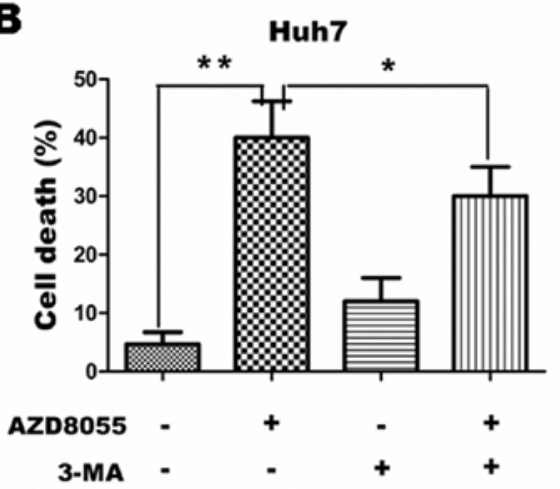

Hep3B

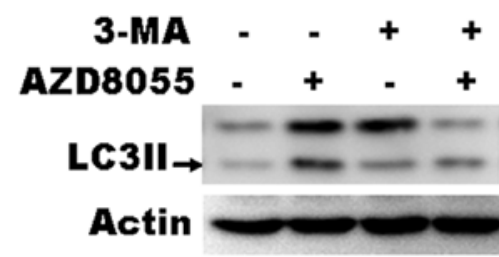

*
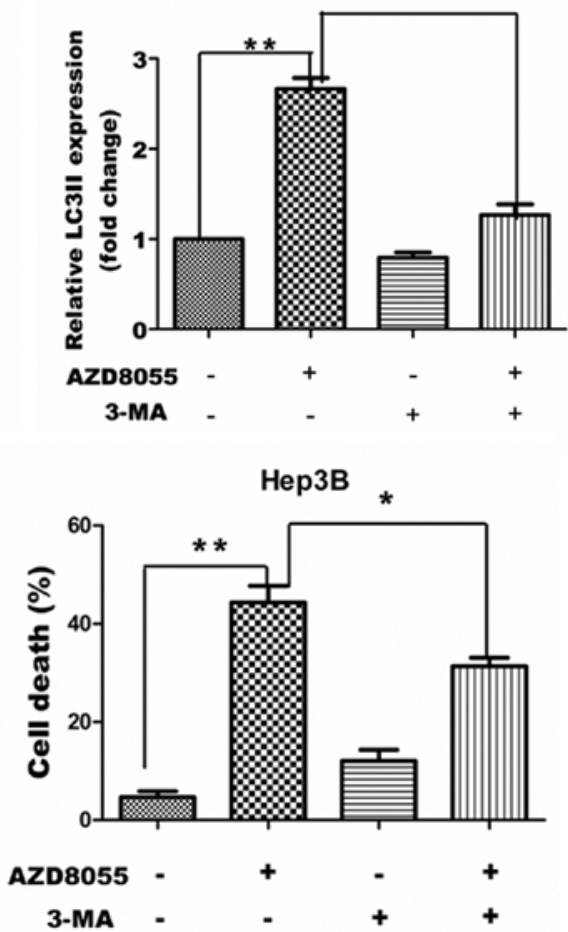

Figure 3. Effects of 3-MA on AZD8055-induced cell death in HCC cells. (A) 3-MA decreases LC3-II expression in Huh7 and Hep3B cells. Cells were treated with DMSO or $100 \mathrm{nM}$ AZD8055 for $12 \mathrm{~h}$ following 2-h pretreatment of $2 \mathrm{mM} 3$-MA. After treatment, cell extracts were prepared for immunoblot analysis. The levels of LC3-II were detected using the corresponding antibodies. $\beta$-actin was used as a loading control (left). After normalization to $\beta$-actin, the relative levels of LC3-II were compared with those at time 0 as a value of one. Data represent mean \pm SD of 3 independent experiments (right). (B) Inhibition of autophagy by 3-MA decreases cell death in Huh7 and Hep3B cells. Cells were treated with DMSO or $100 \mathrm{nM}$ AZD8055 for $12 \mathrm{~h}$ following 2-h pretreatment of 2 mM 3-MA. Cell death was assessed by trypan blue staining. Data represent the mean \pm SD derived from at least 3 separate experiments. ${ }^{*} \mathrm{P}<0.05$, ${ }^{* *} \mathrm{P}<0.01$.

To further verify an increase in autophagic markers induced by AZD8055, we assessed the effects of AZD8055 on the levels of Atg-5/12, a protein complex formed by activation of autophagy $(14,15)$, BECN1 and on the conversion of the cytoplasmic form of LC3-I protein $(18 \mathrm{kDa})$ to the preautophagosomal and autophagosomal membrane-bound form of LC3-II (16 kDa). As shown in Fig. 2B, immunoblot analysis revealed that AZD8055 treatment led to a time-dependent increase in the levels of conjugated Atg-5, BECN1 and LC3-II proteins in both tested cell lines. Quantitative analysis indicated that AZD8055 treatment led to an increase in Atg-5/12, BECN1 and LC3-II levels as early as $12 \mathrm{~h}$ after drug exposure, gradually increasing thereafter (Fig. 2B). Collectively, these data suggest that AZD8055 induces autophagy in HCC cells.

Inhibition of autophagy by 3-methyladenine decreases AZD8055-induced cell death in HCC cells. To confirm the contribution of the increase in the autophagic compartment to AZD8055-induced cell death, we analyzed the effect of 3-methyladenine (3-MA), a well known inhibitor of autophagosome formation (16). Huh7 and Hep3B cells were co-treated with $2 \mathrm{mM} 3-\mathrm{MA}$ and $50 \mathrm{nM}$ AZD8055 for $12 \mathrm{~h}$ and then cell pellets were harvested to determine the amount of the autophagic marker LC3-II. As expected, AZD8055 treatment resulted in increased levels of LC3-II in Huh7 and Hep3B cells. 3-MA treatment led to a significant decrease in AZD8055-induced LC3-II expression in both tested cell lines (Fig. 3A). In addition, we tested whether co-treatment with 3-MA could affect AZD8055-induced cell death. As shown in Fig. 3B, co-treatment with 2 mM 3-MA resulted in a partial but significant inhibition of AZD8055-induced cell death, as measured by MTT assay, in Huh7 and Hep3B cells. The fact that chemical blockage of autophagosome formation reduced cell death confirmed that the observed accumulation of autophagosomes in cells treated with this drug contributes, at least in part, to its cellular toxicity.

Role of AMPK activation in AZD8055-induced autophagic cell death in HCC cells. Previous studies have demonstrated the crucial role of AMP-dependent protein kinase (AMPK) in regulation of autophagy. Thus, we tested whether AZD8055induced cell death was associated with the activation of AMPK 
A
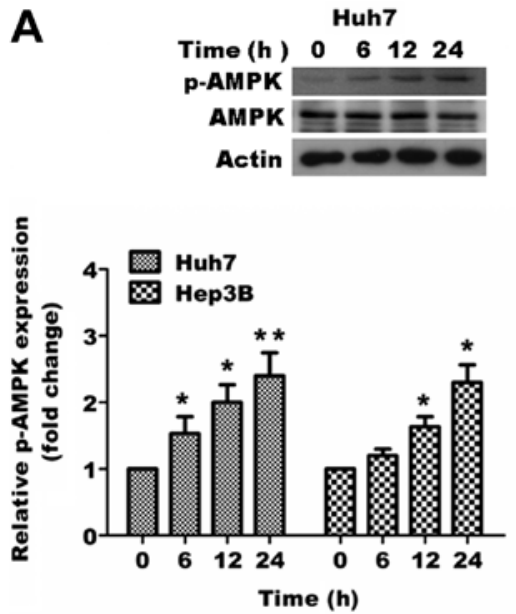

B

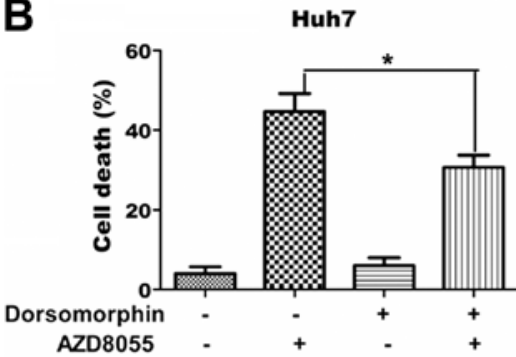

Hep3B

Time (h) $0 \quad 61224$

P-AMPK

AMPK

Actin
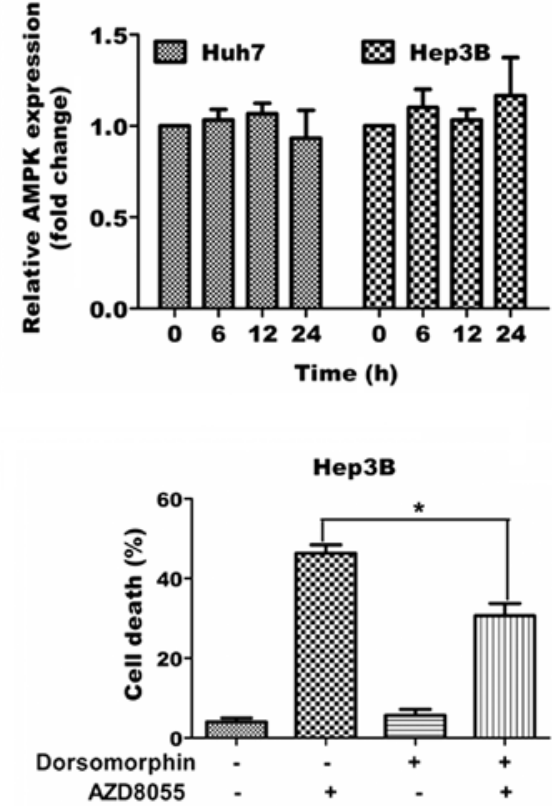

C
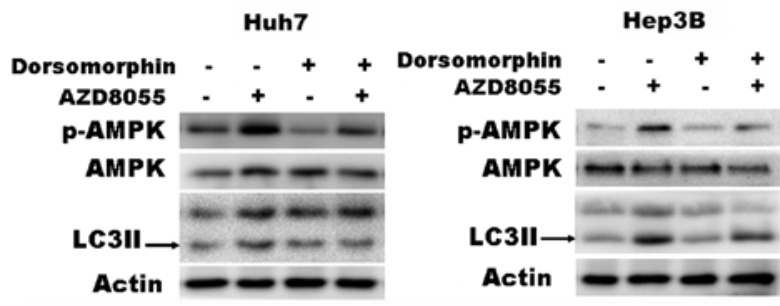

Huh7
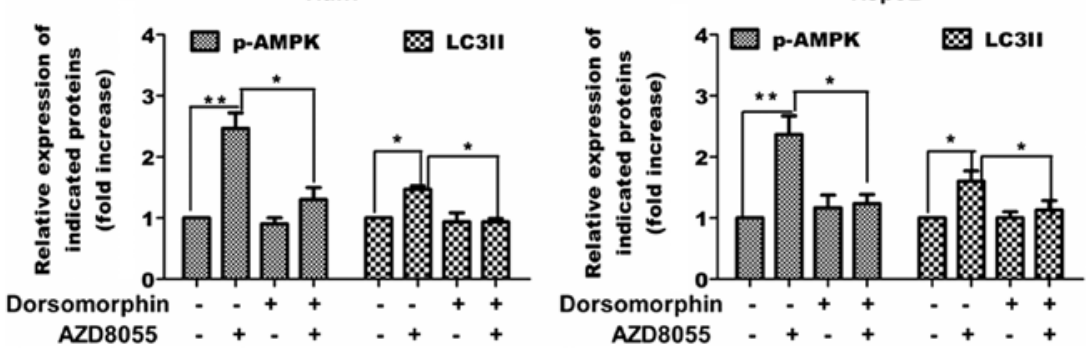

Figure 4. Role of AMPK activation in AZD8055-induced cell death. (A) Cells were treated with $100 \mathrm{nM} \mathrm{AZD8055} \mathrm{for} \mathrm{the} \mathrm{indicated} \mathrm{times.} \mathrm{After} \mathrm{treatment,}$ cells were harvested and cell extracts were prepared for immunoblot analysis. The levels of AMPK and p-AMPK were detected by immunoblot analysis using the corresponding antibodies. $\beta$-actin was used as a loading control. The amount of AMPK and p-AMPK was quantified by a laser densitometer. After normalization to $\beta$-actin, the relative levels of AMPK and p-AMPK were compared with those at time 0 as a value of one. Data represent mean \pm SD of 3 independent experiments (right). ${ }^{*} \mathrm{P}<0.05,{ }^{* *} \mathrm{P}<0.01$. (B) Blocking AMPK activation by dorsomorphin increases cell death in Huh7 and Hep3B cells. Cells were treated with DMSO or $100 \mathrm{nM}$ AZD8055 for $12 \mathrm{~h}$ following 2-h pretreatment of $2 \mathrm{mM}$ dorsomorphin. Cell death was assessed by trypan blue staining. Data represent the mean \pm SD derived from at least 3 separate experiments. (C) Blocking AMPK activation by dorsomorphin decreases LC3-II expression in Huh7 and Hep3B cells. Cells were treated with DMSO or $100 \mathrm{nM}$ AZD8055 for $12 \mathrm{~h}$ following 2-h pretreatment of $2 \mathrm{mM}$ dorsomorphin. After treatment, cell extracts were prepared for immunoblot analysis. The levels of LC3-II were detected using the corresponding antibodies. $\beta$-actin was used as a loading control (left). Data represent means $\pm \mathrm{SD}$ of 3 independent experiments (right). ${ }^{*} \mathrm{P}<0.05,{ }^{* *} \mathrm{P}<0.01$.

in Huh7 cells. Initially, we tested the effect of AZD8055 on activation of AMPK and found AZD8055 caused increased phosphorylation of AMPK (Fig. 4A). Next we blocked activation of AMPK by using AMPK inhibitor dorsomorphin and tested whether co-treatment with dorsomorphin affected
AZD8055-induced cell death and the amount of autophagic marker LC3-II. As shown in Fig. 4B and C, blocking activation of AMPK caused significant attenuation of AZD8055-induced cell death as measured by MTT assay and expression of LC3-II as measured by western blot analysis. 


\section{Discussion}

Hepatocellular carcinoma (HCC) is one of the most common malignant tumors with steadily increasing incidence and mortality rates in various countries (1). HCC is widely regarded as a chemotherapy-resistant disease. These drawbacks necessitate the continued search for novel therapeutic strategies for HCC.

Autophagy is an evolutionarily conserved process in which cellular organelles and macromolecules are degraded for recycling of bioenergetic components (17). As an essential mechanism controlling cellular homeostasis, autophagy is intimately associated with the regulation of cell survival and cell death. Often a stress or injury signal could activate both the cell death and autophagy pathways, in which the role of autophagy could vary depending on the context. In particular, the role of autophagy in liver cancer biology/therapy remains unclear. A previous study demonstrated a dual role of autophagy in cancer therapy (18); autophagy may suppress tumorigenesis or it may provide cancer cells with a rescue mechanism under unfavorable conditions.

Autophagy could serve as novel mechanism to kill cancer cells. Autophagy is also referred to as programmed cell death type II, as opposed to apoptosis or programmed cell death type I $(19,20)$. Recently, inducing autophagic cell death became a novel treatment strategy for cancer therapy. Several chemotherapeutic agents have been observed for inducing autophagic cell death in various tumor cells $(21,22)$. In the present study, we investigated the potential of AZD8055 as a therapeutic agent in HCC partially through the perspective of inducing autophagic cell death.

AZD8055 is a specific mammalian target of rapamycin kinase inhibitor with profound growth inhibitory effects in cervical (3), lung (4), breast cancer (4), glioblastoma (4) and osteosarcoma (4) cells and antitumor activity in vivo (4). The effects of AZD8055 on HCC cells has not been studied. We reported that AZD8055 induced cell death associated with autophagic features in HCC cells, as demonstrated by increased autophagy-related protein. The induction of autophagy seems to be indispensable for inhibitory effects of AZD8055, as demonstrated by the fact that inhibition of autophagy by 3-MA attenuates AZD8055-induced cell death in Huh7 and Hep3B cells.

A better understanding of the mechanism underlying AZD8055-mediated autophagy is critical for the development of novel therapies. We further investigated the mechanism underlying AZD8055-mediated autophagy in HCC. AZD8055 is an ATP-competitive mTOR kinase inhibitor (4). The inhibitory effect of AZD8055 is closely related to ATP levels (4). AMPK acts as a major sensor of cellular energy status in cancer and is critically involved in the efficiency of anticancer agents (23-27). We observed that AZD8055 caused AMPK activation in HCC cells, which has been proved to modulate the induction of autophagy under low energy conditions by dual regulation of mTORC1 and ULK1 $(28,29)$. We found that AZD8055 triggered the activation of AMPK and inhibition of AMPK by dorsomorphin attenuated AZD8055-induced autophagic cell death. The data presented here indicate the key role of AMPK activation in AZD8055-induced autophagic cell death.
In the present study, we investigated the potential of AZD8055 as a therapeutic agent in HCC. We reported that AZD8055 induces cell death associated with autophagic features and APMK activation. Our findings provide a better understanding of the molecular mechanisms underlying the inhibitory effects of AZD8055 and demonstrate the potential of AZD8055 as a therapeutic agent for HCC.

\section{Acknowledgements}

The authors thank Dr Qitao Yan (Southern Medical University) for his technical assistance. The present study was supported by grants from the Science and Technology Program of Guangdong Province (2008B030301028) and the Science and Technology Innovation Fund of Guangdong Medical College (STIF201107).

\section{References}

1. Sherman M: Epidemiology of hepatocellular carcinoma. Oncology 78 (Suppl 1): 7-10, 2010.

2. Tanaka M, Katayama F, Kato H, et al: Hepatitis B and C virus infection and hepatocellular carcinoma in China: a review of epidemiology and control measures. J Epidemiol 21: 401-416, 2011.

3. Li S, Li Y, Hu R, et al: The mTOR inhibitor AZD8055 inhibits proliferation and glycolysis in cervical cancer cells. Oncol Lett 5: 717-721, 2013.

4. Chresta CM, Davies BR, Hickson I, et al: AZD8055 is a potent, selective, and orally bioavailable ATP-competitive mammalian target of rapamycin kinase inhibitor with in vitro and in vivo antitumor activity. Cancer Res 70: 288-298, 2010.

5. Holt SV, Logie A, Davies BR, et al: Enhanced apoptosis and tumor growth suppression elicited by combination of MEK (selumetinib) and mTOR kinase inhibitors (AZD8055). Cancer Res 72: 1804-1813, 2012.

6. Levine B and Klionsky DJ: Development by self-digestion: molecular mechanisms and biological functions of autophagy. Dev Cell 6: 463-477, 2004.

7. Huang S, Yang ZJ, Yu C and Sinicrope FA: Inhibition of mTOR kinase by AZD 8055 can antagonize chemotherapy-induced cell death through autophagy induction and down-regulation of p62/ sequestosome 1. J Biol Chem 286: 40002-40012, 2011.

8. Sini P, James D, Chresta C and Guichard S: Simultaneous inhibition of mTORC1 and mTORC2 by mTOR kinase inhibitor AZD8055 induces autophagy and cell death in cancer cells. Autophagy 6: 553-554, 2010.

9. Willems L, Chapuis N, Puissant A, et al: The dual mTORC1 and mTORC2 inhibitor AZD8055 has anti-tumor activity in acute myeloid leukemia. Leukemia 26: 1195-1202, 2012.

10. Huang HL, Zheng WL, Zhao R, Zhang B and Ma WL: FBXO31 is down-regulated and may function as a tumor suppressor in hepatocellular carcinoma. Oncol Rep 24: 715-720, 2010.

11. Strober W: Trypan blue exclusion test of cell viability. Curr Protoc Immunol Appendix 3: Appendix 3B, 2001.

12. Zhao R, Yan Q, Lv J, et al: CHD5, a tumor suppressor that is epigenetically silenced in lung cancer. Lung Cancer 76: 324-331, 2012.

13. Aoki H, Kondo Y, Aldape K, et al: Monitoring autophagy in glioblastoma with antibody against isoform B of human microtubule-associated protein 1 light chain 3. Autophagy 4: 467-475, 2008.

14. Matsushita M, Suzuki NN, Obara K, Fujioka Y, Ohsumi Y and Inagaki F: Structure of Atg5.Atg16, a complex essential for autophagy. J Biol Chem 282: 6763-6772, 2007.

15. Pyo JO, Jang MH, Kwon YK, et al: Essential roles of Atg5 and FADD in autophagic cell death: dissection of autophagic cell death into vacuole formation and cell death. J Biol Chem 280: 20722-20729, 2005.

16. Seglen PO and Gordon PB: 3-Methyladenine: specific inhibitor of autophagic/lysosomal protein degradation in isolated rat hepatocytes. Proc Natl Acad Sci USA 79: 1889-1892, 1982.

17. Wang CW and Klionsky DJ: The molecular mechanism of autophagy. Mol Med 9: 65-76, 2003. 
18. Kondo Y, Kanzawa T, Sawaya R and Kondo S: The role of autophagy in cancer development and response to therapy. Nat Rev Cancer 5: 726-734, 2005.

19. Bursch W, Hochegger K, Torok L, Marian B, Ellinger A and Hermann RS: Autophagic and apoptotic types of programmed cell death exhibit different fates of cytoskeletal filaments. J Cell Sci 113: 1189-1198, 2000.

20. Bursch W, Ellinger A, Gerner C, Frohwein U and Schulte-Hermann R: Programmed cell death (PCD). Apoptosis, autophagic PCD, or others? Ann NY Acad Sci 926: 1-12, 2000.

21. Le XF, Mao W, Lu Z, Carter BZ and Bast RC Jr: Dasatinib induces autophagic cell death in human ovarian cancer. Cancer 116: 4980-4990, 2010.

22. Xiong HY, Guo XL, Bu XX, et al: Autophagic cell death induced by 5-FU in Bax or PUMA deficient human colon cancer cell. Cancer Lett 288: 68-74, 2010.

23. Fruman DA and Edinger AL: Cancer therapy: staying current with AMPK. Biochem J 412: e3-e5, 2008.

24. Ji C, Yang B, Yang YL, et al: Exogenous cell-permeable C6 ceramide sensitizes multiple cancer cell lines to Doxorubicininduced apoptosis by promoting AMPK activation and mTORC1 inhibition. Oncogene 29: 6557-6568, 2010.
25. Yun SM, Jung JH, Jeong SJ, Sohn EJ, Kim B and Kim SH: Tanshinone IIA induces autophagic cell death via activation of AMPK and ERK and inhibition of mTOR and p70 S6K in KBM-5 leukemia cells. Phytother Res: Jun 27, 2013 (Epub ahead of print). doi: 10.1002/ptr.5015.

26. Puissant A, Robert G, Fenouille N, et al: Resveratrol promotes autophagic cell death in chronic myelogenous leukemia cells via JNK-mediated p62/SQSTM1 expression and AMPK activation. Cancer Res 70: 1042-1052, 2010.

27. Chandrasekar B, Boylston WH, Venkatachalam K, Webster NJ, Prabhu SD and Valente AJ: Adiponectin blocks interleukin18-mediated endothelial cell death via APPL1-dependent AMP-activated protein kinase (AMPK) activation and IKK/NF-кB/PTEN suppression. J Biol Chem 283: 24889-24898, 2008.

28. Lee JW, Park S, Takahashi Y and Wang HG: The association of AMPK with ULK1 regulates autophagy. PLoS One 5: e15394, 2010.

29. Egan DF, Shackelford DB, Mihaylova MM, et al: Phosphorylation of ULK1 (hATG1) by AMP-activated protein kinase connects energy sensing to mitophagy. Science 331: 456-461, 2011. 